\title{
Was ist «Urban Forestry»?
}

Cecil C. Konijnendijk van den Bosch, University of British Columbia (CA)*, interviewt von Marco Pütz**

\begin{abstract}
Diese Frage loten wir in einem Interview mit Cecil C. Konijnendijk van den Bosch aus. C. Konijnendijk ist eine prägende Persönlichkeit der internationalen Urban-Forestry-Community. Er lehrt, forscht und publiziert seit über 20 Jahren zur Rolle von Bäumen, Wäldern und Grünräumen in Städten. Seit 2016 ist er Professor für Urban Forestry an der University of British Columbia in Vancouver (CA), davor war er Professor für Green Space Management an der Universität Kopenhagen (DK). C. Konijnendijk hat Forest Policy an der Universität Wageningen (NL) studiert und in Forest Policy and Economics an der Universität Joensuu (FI) promoviert.

doi: $10.3188 /$ szf.2017.0242

* Department of Forest Resources Management, CA-Vancouver, BC V6T 1Z4, E-Mail cecil.konijnendijk@ubc.ca

** Eidgenössische Forschungsanstalt WSL, Zürcherstrasse 111, CH-8903 Birmensdorf, E-Mail marco.puetz@wsl.ch
\end{abstract}

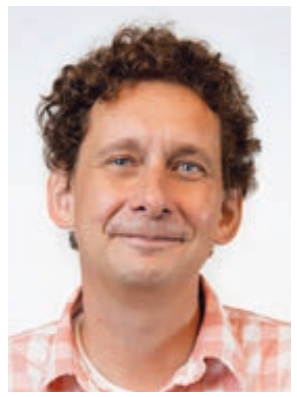

Foto: Jamie Myers
Das Europäische Forum zu Urban Forestry (EFUF) feiert 2017 sein 20-jähriges Bestehen. Diese Konferenzreihe ist als führendes Forum für die Urban-Forestry-Community bekannt. Was war vor 20 Jahren im Hinblick auf Themen und Teilnehmende anders?

Cecil C. Konijnendijk van den Bosch (CKB): Anfangs lag der Schwerpunkt des Forums stärker auf der Forstwirtschaft und nicht auf der weiter gefassten Ressource «Urban Forest/Grünraum». Im Laufe der Jahre gewannen dann Aspekte wie beispielsweise die Baumzucht an Bedeutung. Bei den ersten Foren in Wuppertal (DE) und Aarhus (DK) stellten zudem Praktiker und Praktikerinnen die grösste Teilnehmergruppe neben nur einer Handvoll Forschenden. Und schliesslich kamen die meisten Teilnehmenden aus Ländern Mitteleuropas. Englisch und Deutsch waren die beiden Sprachen, die auf dem ersten EFUF in Wuppertal gesprochen wurden.

Die Urban-Forestry-Debatte begann vor einigen Jahrzehnten in den USA, in Kanada, Australien und Grossbritannien und hat sich seither über die ganze Welt ausgebreitet. Welches sind die grossen Unterschiede zwischen Kontinenten und Ländern beim Verständnis und bei der Bedeutung von Urban Forestry? Was könnte Europa von anderen Kontinenten lernen?

$C K B$ : In Nordamerika hatte Urban Forestry eine stärkere Ausrichtung auf Strassenbäume, wenngleich sich das in jüngster Zeit geändert hat, beispielsweise in Kanada. Ich würde sagen, dass Urban Forestry und die damit verbundene Forschung in Nordamerika eine stärkere naturwissenschaftliche Komponente hatte, wobei der Schwerpunkt auf dem Umgang mit Baumschädlingen und -krankheiten, dem Beschnitt, aber auch auf den Ökosystemleistungen der Stadtbäume lag (z.B. Regenwassermanagement, Energieeinsparungen). Insbesondere in den USA hat die Forschung nach Wegen gesucht, einige dieser Vorteile zu quantifizieren, auch in finanzieller Hinsicht, um bessere Argumente für Urban Forestry gegenüber Entscheidungsträgern zu gewinnen. Grossbritannien und Australien sind dieser Tradition zum Teil gefolgt, aber vor allem in Grossbritannien - wie im übrigen Europa legte man einen stärkeren Fokus auf den Wald sowie auf die gesellschaftlichen Werte von Urban Forests.

In Europa können wir definitiv von den USA lernen, wenn es um eine solide Bewertung von Urban Forests und ihrer Leistungen geht. Auch im Hinblick auf die Entwicklung von Partnerschaften, mit staatlichen Behörden bis hin zu Nichtregierungsorganisationen, können wir von unseren amerikanischen Kolleginnen und Kollegen lernen. Und wir müssen auch ein wenig neidisch sein auf die Bundesprogramme der USA für Urban Forestry und die dort zur Verfügung gestellten Finanzmittel.

Meine amerikanischen Kollegen sagen mir allerdings auch, wie sehr beeindruckt sie sind von dem weiten Feld und der Vielseitigkeit der europäischen Forschung im Bereich Urban Forestry. Sie schätzen vor allem die sozialwissenschaftlichen Arbeiten und den Fokus auf menschliche Gesundheit und menschliches Wohlbefinden. 
Welches sind derzeit die bedeutendsten Trends in Forschung und Praxis im Bereich Urban Forestry ganz allgemein und speziell in Europa?

$C K B$ : Die Forschung zur Erfassung und Bewertung von Urban Forests hat sich enorm verbessert. Mit Satellitenbildern, LiDAR, Fernerkundung usw. stehen uns wesentlich bessere Hilfsmittel zur Verfügung, um Planung und Management zu unterstützen. Zudem können wir dank den Fortschritten bei den geografischen Informationssystemen räumliche Informationen wesentlich besser darstellen.

Ich würde sagen, dass die Erforschung der Bedeutung des Grünraums für die menschliche Gesundheit ein weiterer Trend ist. Hier haben wir im vergangenen Jahrzehnt wirklich grosse Fortschritte gemacht, und weitere Fortschritte sind zu erwarten. Die Notwendigkeit, die Städte an den Klimawandel anzupassen, hat ebenfalls viele neue Forschungsarbeiten ausgelöst, zum Beispiel zur Rolle von Grünflächen bei der Kühlung unserer Städte und als Puffer für die Auswirkungen des Klimawandels (z.B. Überschwemmungen, Hitzewellen). Mit dem Aufkommen von Konzepten und Ansätzen wie «grüne Infrastruktur» oder «naturbasierte Lösungen» wurde auch stärker auf die Zusammenhänge zwischen Stadtplanung, städtischer Nachhaltigkeit/Resilienz sowie den Urban Forests geachtet.

Urban Forestry ist ein recht neuer Begriff in der Schweiz, der häufig in der englischen Originalbezeichnung verwendet und nicht auf Deutsch übersetzt wird. Urban Forestry bedeutet mehr als «urbane Forstwirtschaft», also «Forstwirtschaft im städtischen Gebiet». Zudem geht es bei Urban Forestry nicht nur um Wald. Aber worum geht es bei Urban Forestry eigentlich?

$C K B$ : Für mich geht es bei Urban Forestry im Grunde ums Integrieren, um das Denken und Arbeiten über traditionelle Grenzen hinweg. Bäume und Wälder sind das vereinende Element, das die verschiedenen Sektoren, Interessen und Fachgebiete zusammenbringt. Wir haben alle unterschiedliche Hintergründe, aber wir befassen uns alle mit Bäumen und den Beziehungen zwischen Menschen und Bäumen, zwischen Städten und Wäldern. Ursprünglich komme ich aus der Forstwirtschaft, mein Arbeitsfeld reicht jedoch vom Einzelbaum im privaten Garten bis zum grossräumigen Waldeinzugsgebiet. Für mich ist es das, was Urban Forestry ausmacht - und mir Freude bereitet!

Was ist Urban Forestry? Ein Begriff, ein Konzept, eine Theorie, ein Paradigma, eine Philosophie, ein Modewort? Ein Versuch von Planern,

Architekten, Umweltschützern oder anderen, den Wald für sich zu vereinnahmen?

$C K B$ : Urban Forestry ist ein interdisziplinäres Feld, das Grenzen überwinden will und nach Ver- besserungen beim Planen, Gestalten und Managen von Natur und Bäumen im urbanen Gebiet sucht. Es gibt kein einzelnes Fachgebiet oder einen Beruf, die Urban Forestry für sich beanspruchen könnten. Wir haben alle etwas beizutragen. Für die Forstwirtschaft war Urban Forestry sehr wichtig, indem sie ihr gezeigt hat, dass sie auch ein urbanes Mandat hat. In gewisser Weise ist Urban Forestry vorwiegend eine Einstellung, eine Denkweise und eine andere Sprache, die mehr integriert als trennt.

Welches sind die bedeutendsten aktuellen politischen Entwicklungen und Programme im Bereich Urban Forestry in Europa und international?

$C K B$ : Die USA sind das Land mit den fortschrittlichsten nationalen Programmen für Urban Forestry, mit entsprechenden politischen Vorgaben und Finanzierungsquellen. Andere Länder, etwa China, haben begonnen, diesem Beispiel zu folgen. In Europa ist Grossbritannien bei der Integration von Urban Forestry in die nationale Politik wahrscheinlich am weitesten. In den meisten anderen Ländern ist Urban Forestry noch weitgehend den Städten selbst überlassen, ohne regionale oder nationale Koordination.

Die Ernährungs- und Landwirtschaftsorganisation der Vereinten Nationen (FAO) verdient ein Lob für ihre weltweite Arbeit zu Urban und Peri-urban Forestry. So hat die FAO beispielsweise kürzlich einen umfassenden Leitfaden für Urban Forestry herausgeben (Salbitano et al 2016). Auch auf lokaler Ebene findet man einige Beispiele. Als sehr gutes Beispiel würde ich Melbourne (AU) erwähnen, aber auch Städte in Nordamerika, Asien und Europa lassen sich als Bespiele anführen.

Welche Rahmenkonzepte und Methoden stehen zur Verfügung, um vergleichende Forschung zu Urban Forestry zu betreiben?

$C K B$ : Ich würde sagen, dass i-Tree ${ }^{1}$, eine Sammlung von Software und Modellen, die vom US Forest Service entwickelt wurde, zurzeit das am weitesten verbreitete Hilfsmittel ist und den Rahmen vorgibt. Analysen der Baumkronenbedeckung in der Stadt und des Nutzens von Urban Forests mit i-Tree wurden auf der ganzen Welt durchgeführt.

Aber es gibt auch andere Bereiche, zum Beispiel vergleichende Studien zur Governance von Urban Forests und natürlich zur Pflege von Stadtbäumen. Die International Society of Arboriculture ${ }^{2}$ fördert schon seit vielen Jahren gute Verfahren in der Baumzucht.

Welche Arten von Hilfsmitteln und Instrumenten stehen zur Verfügung, die in der Praxis eingesetzt und angepasst werden können?

1 www.itreetools.org (8.2.2017)

2 www.isa-arbor.com (8.2.2017) 
Abb 1 Einblick in den Sonsbeek Park in Arnhem (NL), den Lieb-

lings-Urban-Forest von C. Konijnendijk. Foto: Sophie Molendijk

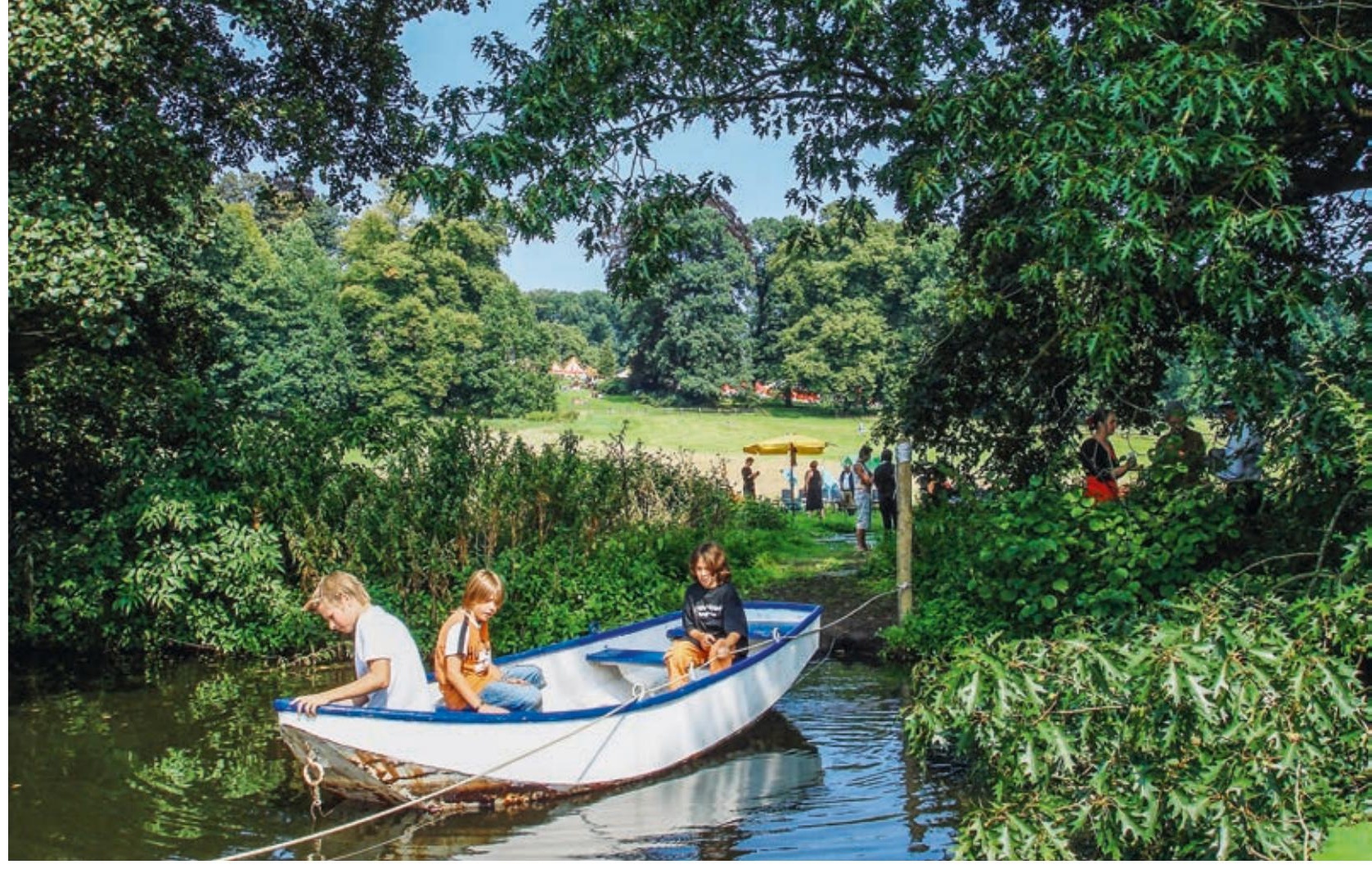

$C K B$ : Es gibt viele solche Hilfsmittel, wie oben zum Teil schon ausgeführt. Auf höherer Skala haben wir i-Tree und eine Reihe von Tools, die auf Fernerkundung und LiDAR basieren. Stetig wurde auch für Weiterentwicklungen im Bereich der Bauminventuren sowie der Pflanzung und der Pflege von Bäumen gesorgt. Moderne Technologien haben die Erfassung und das Monitoring von Baumbeständen erheblich vereinfacht, und GIS kann alle Informationen auf wesentlich verständlichere Weise zusammenbringen.

Welches sind die Kernkompetenzen, in denen Urban Foresters geschult werden sollten?

$C K B$ : Das ist eine wichtige Frage, über die wir in unserem neuen Bachelorprogramm von Urban Forestry hier an der University of British Columbia sehr viel diskutieren. Urban Foresters sollten natürlich über Bäume und Wälder Bescheid wissen, und sie sollten recht viel darüber wissen, wie Bäume wachsen oder welche Herausforderungen sich aus den Wachstumsbedingungen in der Stadt ergeben. Aber Urban Forestry sollte auch eine starke gesellschaftliche Dimension haben, die dazu beiträgt, die vielen Beziehungen zwischen Menschen und Bäumen zu verstehen, und es ermöglicht, die Bevölkerung in Entscheidungsprozesse mit einzubeziehen. Urban Foresters sollten sehr gute Kommunikationsfähigkeiten besitzen und wissen, wie man im politischen Umfeld agiert. Sie sollten auch in grösseren sozioökologischen Systemzusammenhängen denken können. Ebenso müssen sie viele unterschiedliche Informationen verstehen und für die Entscheidungsfindung verarbeiten können. Und sie müssen mit Fachleuten aus unterschiedlichen Bereichen zusam- menarbeiten können - von Planung und Ökologie bis hin zur öffentlichen Gesundheit.

Urban Forestry wird oft als integratives Konzept bezeichnet. Was genau muss integriert werden? Und wer ist für diese Integration verantwortlich oder sollte es sein?

$C K B$ : Integration bezieht sich zunächst einmal auf die Notwendigkeit, die verschiedenen Komponenten von Urban Forests zu berücksichtigen, vom Strassenbaum bis zum an die Stadt angrenzenden Wald. Es ist ähnlich wie beim Begriff der grünen Infrastruktur, wobei Urban Forestry stärker baumorientiert sowie praxisbezogener ist. Aber wir sprechen auch über die Integration wissenschaftlicher Fächer und Disziplinen, von Sektoren, Berufen und der Bevölkerung. Die Koordination sollte idealerweise durch einen städtischen Urban Forester erfolgen.

Urban Forsts sind ein zentrales Element im Netzwerk von Grünräumen und ein entscheidender Faktor zur Verbesserung der Lebensqualität in Städten und darum herum. Was können wir tun, um die Urban Forests zu schützen, zu verbessern und auszuweiten?

$C K B$ : Information und gute wissenschaftliche Arbeit halte ich für wichtig, da wir wissen müssen, was wir haben und was die Urban Forests im Hinblick auf Ökosystemleistungen bieten. Wir sollten auch in der Lage sein, gegenüber Entscheidungsträgern überzeugend zu argumentieren. Letzteres erfordert starke Allianzen, mit der Bevölkerung, Interessengruppen und Fachleuten verschiedener Gebiete. Wir müssen gute Praktiken und Beispiele identifizieren, diese fördern und sie in Urban-Forestry-Netzwerken teilen. 
In der Schweiz gehören lokale Forstbehörden und Waldbesitzer zu den wichtigen Interessengruppen von Urban Forestry. Doch ihnen fehlen oft die finanziellen Mittel. Gibt es Beispiele für die Finanzierung von Urban-Forestry-Projekten?

CKB: Die Finanzierung ist immer ein Problem, insbesondere in Zeiten, in denen traditionelle Mittelflüsse gekürzt werden. Aber es gibt verschiedene Möglichkeiten. Dazu gehören beispielsweise nationale Finanzierungsprogramme wie in den USA oder Stadtentwicklungsprogramme, bei welchen nicht nur die Kosten der neuen Wohnungen, sondern auch die Begrünungskosten einberechnet und überwälzt werden. Die Anpassung an den Klimawandel wird grosse Budgets für die Städte notwendig machen. Urban Forestry hat das Potenzial, gewissen Anpassungserfordernissen kostengünstig zu begegnen. Krankenversicherungen könnten Urban-ForestryProgramme finanzieren, die die öffentliche Gesundheit fördern. Wasserwirtschaftsämter oder Versorgungsunternehmen könnten sich an der Finanzierung von Urban Forestry beteiligen, um Trinkwasserressourcen zu sichern. Zu den Beteiligten aus dem privaten Sektor könnten Sponsoren gehören, und sogar «hybride», in öffentlich-privaten Partnerschaften entwickelte Parks sind denkbar.

In der Schweiz kennen wir den Waldentwicklungsplan als grundlegendes strategisches Forstplanungsinstrument. Es gibt keine speziellen Urban-Forestry-Pläne. Welches wären die Vorteile von solchen Plänen und was sollten sie enthalten?

$C K B$ : Das hängt meiner Meinung nach von der Art der bestehenden Konzepte und Planungen ab und von den Möglichkeiten, die sie für die Anpassung an städtische Bedingungen und Bedürfnisse

\section{Qu'appelle-t-on «foresterie urbaine»?}

Nous nous penchons sur cette question lors d'un entretien avec Cecil C. Konijnendijk van den Bosch, professeur de foresterie urbaine à I'Université de Colombie britannique de Vancouver (CA). On entend par foresterie urbaine un champ de travail interdisciplinaire qui aborde l'étude, la conception et l'exploitation d'espaces verts urbains, et plus particulièrement «l'arbre» ou encore «la forêt» au sein de ces espaces verts. Ce domaine prend de plus en plus d'importance en raison de la promotion de la santé, de l'amélioration de la qualité de vie en ville ainsi que de l'adaptation des villes aux changements climatiques. Les programmes de foresterie urbaine doivent être intimement liés à l'aménagement des villes, et présenter une forte dimension sociétale. Au niveau local, on rencontre quelques bons programmes de foresterie urbaine, p.ex. celui de la ville de Melbourne (AU). Des programmes nationaux avec des objectifs et des financements politiques sont encore rares. Ce sont les Etats-Unis qui sont le plus en avance, tandis qu'en Europe, c'est la Grande-Bretagne qui a le plus progressé dans l'intégration de la foresterie urbaine dans la politique nationale. bieten. Spezielle Urban-Forestry-Pläne enthalten eine starke Komponente «natürliche Ressourcen»sowie eine starke Komponente «Mensch», und sie anerkennen die engen Beziehungen zwischen städtischer Natur und städtischer Bevölkerung. Sie sollten auch an die breitere grüne Infrastruktur und an die Stadtplanung anknüpfen.

Zum Schluss noch eine persönliche Frage: Welches ist Ihr liebster Urban Forest und warum?

CKB: Mein liebster Urban Forest ist der Sonsbeek Park in Arnhem in den Niederlanden (Abbildung 1), weil er von einzigartiger Schönheit ist und einen fantastischen Rahmen ganz in der Nähe des Stadtzentrums bietet. Die Topografie und die Kombination aus Park und Wald sind auch wirklich grossartig. Die Verantwortlichen von Sonsbeek sind seit vielen Jahren echte Visionäre, und das kommt im aktuellen Park, in seiner Beliebtheit und den vielen Veranstaltungen und Aktivitäten, die dort jedes Jahr stattfinden, zum Ausdruck. Ich glaube, dass die Tatsache, dass ich Sonsbeek während meines Forstwirtschaftsstudiums in Wageningen oft besucht habe, hier auch eine Rolle spielt. Ich habe aber auch noch andere Favoriten, darunter den Sihlwald in der Nähe von Zürich, den Djurgaarden in Stockholm, den Central Park in New York und den Stanley Park in Vancouver.

Übersetzung aus dem Englischen: Marco Pütz

\section{Literatur}

SALBITANO F, BORELLI S, CONIGLIARO M, CHEN Y (2016) Guidelines on urban and peri-urban forestry. Rome: Food and Agriculture Organization of the United Nations, FAO Forestry Paper 178. $172 \mathrm{p}$.

\section{What is "urban forestry"?}

This question is discussed in an interview with Cecil C. Konijnendijk van den Bosch, Professor of Urban Forestry at the University of British Columbia in Vancouver (CA). Urban forestry is an interdisciplinary field dealing with the planning, design and management of urban green spaces, and in particular with trees and forests as elements of these urban green spaces. Urban forestry is gaining importance because of health promotion policies, the improvement of quality of life in cities, and cities adapting to climate change. Urban forestry programs should be well connected with urban planning and feature a strong social dimension. Some good urban forestry programs can be found at the local level, e.g. in the city of Melbourne (AU). However, national urban forestry programs including political standards and funding are rather rare. In this respect, the most advanced urban forest policies can be found in the US; in Europe, the UK is probably ahead in terms of integrating urban forestry into national policies. 